\title{
Training Aided Frequency Offset Estimation for MIMO OFDM Systems via Polynomial Rooting
}

\author{
Yanxiang Jiang, Xiaohu You, Xiqi Gao \\ National Mobile Communications Research Laboratory, \\ Southeast University, Nanjing 210096, China. \\ E-mail: \{yxjiang, xhyu, xqgao\}@seu.edu.cn
}

\author{
Hlaing Minn \\ Department of Electrical Engineering, \\ University of Texas at Dallas, TX 75083-0688, USA. \\ E-mail: hlaing.minn@utdallas.edu
}

\begin{abstract}
In this paper, we investigate training aided frequency offset estimation for multiple-input multiple-output (MIMO) orthogonal frequency division multiplexing (OFDM) systems via polynomial rooting over frequency selective fading channels. By designing the training sequences properly, both integer and fractional carrier frequency offsets (CFO) can be estimated from the polynomials corresponding to the cost function. Moreover, we show through analysis that rooting the cost function is equivalent to rooting the first-order derivative of the cost function in the considered MIMO case. Simulation results verify the good performance of our new CFO estimator and the corresponding analytical results.
\end{abstract}

\section{INTRODUCTION}

Orthogonal frequency division multiplexing (OFDM) is a leading modulation technique for wide-band wireless communications. Combining it with multiple-input multiple-output (MIMO) multi-antenna technique promises a significant increase in the practically achievable throughput over wireless media. The performance of OFDM systems, however, is sensitive to carrier frequency offset (CFO) caused by Doppler effect or the mismatch between transmitter and receiver oscillators [1]. Accurate estimation and compensation of CFO is therefore very important in order to realize the advantages of MIMOOFDM.

CFO estimation is a well-studied problem for single antenna OFDM systems [2]-[8], but a relatively new one for MIMO or MIMO OFDM systems [9]-[14]. Numerical calculations of the CFO estimators in [10] [11] required a large point discrete Fourier transform (DFT) operation and a time consuming line search. To reduce complexity, computationally efficient CFO estimators were introduced in [12]-[14]. Especially, for the training aided CFO estimators in [13] [14], integer CFO (ICFO) was estimated firstly through an $N$-point DFT operation where $N$ is the total number of the subcarriers, and then fractional CFO (FCFO) was estimated through the roots of a complex or real polynomial corresponding to the cost function. Besides, it has been shown recently in [15] that the CFO estimation via polynomial rooting indirectly from the first-order derivative of the cost function outperformed that via polynomial rooting directly from the cost function.

In this paper, based on the CFO estimators in [13] [14] and by designing the training sequences properly, we propose to estimate ICFO and FCFO through the roots of the corresponding complex or real polynomial directly, and thus without the need of the $N$-point DFT operation. Moreover, we reveal the relations between the polynomials corresponding to the cost function and their first-order derivatives, and show that the direct rooting approach is superior to the derivative rooting approach for our considered MIMO case, which is quite different from the blind single antenna case as shown in [15].

The rest of this paper is organized as follows. In Section II, we give the signal model. The new training aided CFO estimator is presented in Section III. Also included in Section III are the analysis of the direct rooting approach and the derivative rooting approach and the analysis of the computational complexity. Simulation results are shown in Section IV. Final conclusions are drawn in Section V.

Notations: Upper (lower) bold-face letters are used for matrices (column vectors). Superscripts $*, T$ and $H$ denote conjugate, transpose and Hermitian transpose, respectively. $(\cdot)_{P}$ denotes the remainder of the number within the brackets modulo $P . \mathfrak{R}(\cdot)$ and $\mathfrak{I}(\cdot)$ denote the real and imaginary parts of the enclosed parameters, respectively. $\otimes$ and $\mathrm{E}(\cdot)$ denote the Kronecker product and expectation operators, respectively. $[\boldsymbol{x}]_{m}$ denotes the $m$-th entry of a column vector $\boldsymbol{x} . \boldsymbol{x}^{(m)}$ denotes the $m$-cyclic-down-shift version of $\boldsymbol{x}$ with $m>0 . \operatorname{diag}\{\boldsymbol{x}\}$ denotes a diagonal matrix with the elements of $\boldsymbol{x}$ on its diagonal. $[\boldsymbol{X}]_{m, n}$ denotes the $(m, n)$-th entry of a matrix $\boldsymbol{X} . \boldsymbol{F}_{N}$ and $\boldsymbol{I}_{N}$ denote the $N \times N$ unitary DFT matrix and the $N \times N$ identity matrix, respectively. $\boldsymbol{e}_{N}^{k}$ denotes the $k$-th column vector of $\boldsymbol{I}_{N} . \mathbf{1}_{Q}\left(\mathbf{0}_{Q}\right)$ and $\mathbf{0}_{P \times Q}$ denote the $Q \times 1$ all-one (all-zero) vector and $P \times Q$ all-zero matrix, respectively. Unless otherwise stated, we assume $(N)_{2 P}=0, Q=N / P, 0 \leq p \leq P-1$, $0 \leq q \leq Q-1,0 \leq \mu \leq N_{t}-1$ and $0 \leq v \leq N_{r}-1$.

\section{Signal Model}

We consider a MIMO OFDM system with $N$ subcarriers, $N_{t}$ transmit antennas and $N_{r}$ receive antennas. Let

$$
\begin{gathered}
M=\operatorname{floor}\left(P / N_{I}\right), N_{I} \geq N_{t}, \\
0 \leq i_{0}<i_{1}<\cdots<i_{\mu}<\cdots<i_{N_{t}-1}<Q .
\end{gathered}
$$

Define $\boldsymbol{\Theta}_{q}=\left[\boldsymbol{e}_{N}^{q}, \boldsymbol{e}_{N}^{q+Q}, \cdots, \boldsymbol{e}_{N}^{q+(P-1)}\right]$. Let $\boldsymbol{s}$ denote a length$P$ Chu sequence [16]. Define $\tilde{\boldsymbol{s}}_{\mu}=\sqrt{Q / N_{t}} \boldsymbol{F}_{P} \boldsymbol{S}^{(\mu M)}$. Then, the training vector at the $\mu$-th transmit antenna is constructed as follows [13] [14]

$$
\tilde{\boldsymbol{t}}_{\mu}=\boldsymbol{\Theta}_{i_{\mu}} \tilde{\mathbf{s}}_{\mu}
$$


For convenience, we henceforth refer to $\left\{\tilde{\boldsymbol{t}}_{\mu}\right\}_{\mu=0}^{N_{t}-1}$ as the Chu sequence based training sequences (CBTS).

Assume that all the transmit-receive antenna pairs are affected by the same CFO. Define

$$
\boldsymbol{D}_{\bar{N}}(\varepsilon)=\operatorname{diag}\left\{\left[1, e^{j 2 \pi \varepsilon / N}, \cdots, e^{j 2 \pi \varepsilon(\bar{N}-1) / N}\right]^{T}\right\},
$$

where $\varepsilon$ is the frequency offset normalized by the subcarrier spacing. Suppose the length- $L$ channel impulse response from the $\mu$-th transmit antenna to the $v$-th receive antenna is denoted by the $L \times 1$ vector $\boldsymbol{h}^{(v, \mu)}$. Define

$$
\tilde{\boldsymbol{h}}^{(v, \mu)}=\boldsymbol{F}_{N}\left[\boldsymbol{e}_{N}^{0}, \boldsymbol{e}_{N}^{1}, \cdots, \boldsymbol{e}_{N}^{L-1}\right] \boldsymbol{h}^{(v, \mu)} .
$$

Then, after removing the cyclic prefix $(\mathrm{CP})$ at the $v$-th receive antenna, the $N \times 1$ received vector $\boldsymbol{y}_{v}$ can be written as [11]

$$
\boldsymbol{y}_{v}=\sqrt{N} e^{j 2 \pi \varepsilon N_{g} / N} \boldsymbol{D}_{N}(\varepsilon) \sum_{\mu=0}^{N_{t}-1}\left\{\boldsymbol{F}_{N}^{H} \operatorname{diag}\left\{\tilde{\boldsymbol{h}}^{(v, \mu)}\right\} \tilde{\boldsymbol{t}}_{\mu}\right\}+\boldsymbol{w}_{v},
$$

where $N_{g}$ denotes the length of the CP and is supposed to be longer than the length of the channel impulse response $L$, $\boldsymbol{w}_{v}$ is an $N \times 1$ vector of additive white complex Gaussian noise (AWGN) samples with zero-mean and equal variance of $\sigma_{w}^{2}$. Let $\boldsymbol{y}=\left[\boldsymbol{y}_{0}^{T}, \boldsymbol{y}_{1}^{T}, \cdots, \boldsymbol{y}_{v}^{T}, \cdots, \boldsymbol{y}_{N_{r}-1}^{T}\right]^{T}$ denote the $N_{r} N \times 1$ cascaded vector from the $N_{r}$ receive antennas. Then, $\boldsymbol{y}$ can be written as [13] [14]

$$
\boldsymbol{y}=\sqrt{N} e^{j 2 \pi \varepsilon N_{g} / N}\left\{\boldsymbol{I}_{N_{r}} \otimes\left[\boldsymbol{D}_{N}(\varepsilon) \boldsymbol{S}\right]\right\} \boldsymbol{h}+\boldsymbol{w},
$$

where

$$
\begin{gathered}
\boldsymbol{h}=\left[\boldsymbol{h}_{0}^{T}, \boldsymbol{h}_{1}^{T}, \cdots, \boldsymbol{h}_{v}^{T}, \cdots, \boldsymbol{h}_{N_{r}-1}^{T}\right]^{T}, \\
\boldsymbol{h}=\left\{\boldsymbol{1}_{N_{t}}^{T} \otimes \boldsymbol{F}_{N}^{H}\right\} \operatorname{diag}\left\{\left[\tilde{\boldsymbol{t}}_{0}^{T}, \tilde{\boldsymbol{t}}_{1}^{T}, \cdots, \tilde{\boldsymbol{t}}_{N_{t}-1}^{T}\right]^{T}\right\}\left\{\boldsymbol{I}_{N_{t}} \otimes\left[\boldsymbol{F}_{N}\left[\boldsymbol{e}_{N}^{0}, \boldsymbol{e}_{N}^{1}, \cdots, \boldsymbol{e}_{N}^{L-1}\right]\right]\right\} \\
\boldsymbol{w}=\left[\boldsymbol{w}_{0}^{T}, \boldsymbol{w}_{1}^{T}, \cdots, \boldsymbol{w}_{v}^{T}, \cdots, \boldsymbol{w}_{N_{r}-1}^{T}\right]^{T} .
\end{gathered}
$$

\section{TRAining Aided CFO Estimation FOR MIMO OFDM SYSTEMS VIA POLYNOMIAL ROOTING}

\section{A. CFO Estimation for MIMO OFDM via Direct Polynomial Rooting}

By exploiting the periodic property of the training sequences, the received vector $\boldsymbol{y}$ can be stacked into the $Q \times N_{r} P$ matrix $\boldsymbol{Y}=\left[\boldsymbol{Y}_{0}, \boldsymbol{Y}_{1}, \cdots, \boldsymbol{Y}_{v}, \cdots \boldsymbol{Y}_{N_{r}-1}\right]$, where

$$
\left[\boldsymbol{Y}_{v}\right]_{q, p}=\left[\boldsymbol{y}_{v}\right]_{q P+p} .
$$

Let $\beta_{\mu}=\varepsilon+i_{\mu}$. Then, $\boldsymbol{Y}$ can be expressed as follows

$$
\boldsymbol{Y}=\boldsymbol{B} \boldsymbol{X}+\boldsymbol{W}
$$

where

$$
\begin{gathered}
\boldsymbol{B}=\left[\boldsymbol{b}_{0}, \boldsymbol{b}_{1}, \cdots, \boldsymbol{b}_{\mu}, \cdots, \boldsymbol{b}_{N_{t}-1}\right], \\
\boldsymbol{b}_{\mu}=\left[1, e^{j 2 \pi \beta_{\mu} / Q}, \cdots, e^{j 2 \pi \beta_{\mu} q / Q}, \cdots, e^{j 2 \pi \beta_{\mu}(Q-1) / Q}\right]^{T}, \\
\boldsymbol{X}=\left[\boldsymbol{X}_{0}, \boldsymbol{X}_{1}, \cdots, \boldsymbol{X}_{v}, \cdots, \boldsymbol{X}_{N_{r}-1}\right], \\
\boldsymbol{X}_{v}=\left[\boldsymbol{x}^{(v, 0)}, \boldsymbol{x}^{(v, 1)}, \cdots, \boldsymbol{x}^{(v, \mu)}, \cdots, \boldsymbol{x}^{\left(v, N_{t}-1\right)}\right]^{T}, \\
\boldsymbol{x}^{(v, \mu)}=\sqrt{P} e^{j 2 \pi \varepsilon N_{g} / N} \boldsymbol{D}_{P}\left(\beta_{\mu}\right) \boldsymbol{F}_{P}^{H} \operatorname{diag}\left\{\tilde{\boldsymbol{s}}_{\mu}\right\} \boldsymbol{\Theta}_{i_{\mu}}^{T} \tilde{\boldsymbol{h}}^{(v, \mu)},
\end{gathered}
$$

and $\boldsymbol{W}$ is the $Q \times N_{r} P$ matrix generated from $\boldsymbol{w}$ in the same way as $\boldsymbol{Y}$.

Let $\boldsymbol{L}$ denote a $Q \times Q$ unitary column conjugate symmetric matrix. Define

$$
\hat{\boldsymbol{R}}_{\boldsymbol{Y} \boldsymbol{Y}}=\mathfrak{R}\left(\boldsymbol{L}^{H} \boldsymbol{Y} \boldsymbol{Y}^{H} \boldsymbol{L}\right) /\left(N_{r} P\right) .
$$

Let $\boldsymbol{E}_{\boldsymbol{X}}$ denote the $Q \times N_{t}$ real matrix which contains the unitary eigen-vectors spanning the signal subspace of $\hat{\boldsymbol{R}}_{\boldsymbol{Y} \boldsymbol{Y}}$. Define

$$
\begin{gathered}
z=e^{j 2 \pi \beta / Q}, g=\cot (\pi \beta / Q), \\
\boldsymbol{a}(z)=\left[1, z, \cdots, z^{Q-1}\right]^{T}, \boldsymbol{a}(g)=\left[1, g, \cdots, g^{Q-1}\right]^{T} .
\end{gathered}
$$

Then, we can estimate $\left\{\beta_{\mu}\right\}_{\mu=0}^{N_{t}-1}$ through the roots of either a complex-coefficient polynomial or a real-coefficient polynomial [13] [14] as follows

$$
\begin{aligned}
& f^{c}(z)=\boldsymbol{a}^{T}(z) \boldsymbol{A}^{c} \boldsymbol{a}(z)=0, \\
& f^{r}(g)=\boldsymbol{a}^{T}(g) \boldsymbol{A}^{r} \boldsymbol{a}(g)=0,
\end{aligned}
$$

where

$$
\begin{gathered}
\boldsymbol{A}^{c}=\boldsymbol{J} \boldsymbol{L}\left[\boldsymbol{I}_{Q}-\boldsymbol{E}_{\boldsymbol{X}} \boldsymbol{E}_{\boldsymbol{X}}^{T}\right] \boldsymbol{L}^{H}, \\
\boldsymbol{A}^{r}=\boldsymbol{\Phi}^{H} \boldsymbol{L}\left[\boldsymbol{I}_{Q}-\boldsymbol{E}_{\boldsymbol{X}} \boldsymbol{E}_{\boldsymbol{X}}^{T}\right] \boldsymbol{L}^{H} \boldsymbol{\Phi},
\end{gathered}
$$

$\boldsymbol{J}$ denotes the $Q \times Q$ exchange matrix with ones on its antidiagonal and zeros elsewhere, $\boldsymbol{\Phi}$ is the $Q \times Q$ column conjugate symmetric matrix with its element given by

$$
[\boldsymbol{\Phi}]_{q, q^{\prime}}=j^{Q-1-q^{\prime}} \sum_{q^{\prime \prime}=\max \left\{0, q+q^{\prime}-Q+1\right\}}^{\min \left\{q, q^{\prime}\right\}}\left\{C_{q}^{q^{\prime \prime}} C_{Q-1-q}^{q^{\prime}-q^{\prime \prime}}(-1)^{Q-1-q-q^{\prime}+q^{\prime \prime}}\right\},
$$

and $C_{q}^{q^{\prime \prime}}=q ! /\left[\left(q-q^{\prime \prime}\right) ! q^{\prime \prime} !\right]$. Note that $\boldsymbol{\Phi}^{H} \boldsymbol{L}$ is a real matrix due to the column conjugate symmetric property of both $\boldsymbol{\Phi}$ and $\boldsymbol{L}$. Let $\Lambda^{c}(z)$ and $\Lambda^{r}(g)$ denote the polynomials transformed from the cost function. Then, we can readily obtain [13]

$$
\begin{gathered}
\Lambda^{c}(z)=z^{1-Q} \cdot \boldsymbol{a}^{T}(z) \boldsymbol{A}^{c} \boldsymbol{a}(z), \\
\Lambda^{r}(g)=\left(g^{2}+1\right)^{1-Q} \cdot \boldsymbol{a}^{T}(g) \boldsymbol{A}^{r} \boldsymbol{a}(g) .
\end{gathered}
$$

Due to the Hermitian property of $\boldsymbol{J} \boldsymbol{A}^{c}$, we establish from (4) that

$$
f^{c}\left(|z|^{-1} e^{j \arg (z)}\right)=\left(|z|^{-1} e^{j \arg (z)}\right)^{2(Q-1)} \cdot\left(f^{c}(z)\right)^{*} .
$$

Hence, the roots of $f^{c}(z)=0$ are pairwise and in the form of $\left\{z,|z|^{-1} e^{j \arg (z)}\right\}$. Note that the roots of the polynomial equation $f^{c}(z)=0$ always exist no matter noise is absent or present, which is quite different from the analysis in [15]. Due to the real symmetric property of $\boldsymbol{A}^{r}$, we immediately establish that the roots of $f^{r}(g)=0$ are also pairwise and in the form of $\{\mathfrak{R}(g) \pm j \mathfrak{J}(g)\}$, where we have used the relationship $\boldsymbol{\Phi}^{H} \boldsymbol{L}=$ $\boldsymbol{\Phi}^{T} \boldsymbol{L}^{*}$. In the following, the pairwise property of the roots of $f^{c}(z)=0$ and $f^{r}(g)=0$ are exploited for the CFO estimation.

To estimate the CFO, we firstly find the $N_{t}$ pairwise roots of $f^{c}(z)=0$ which are closest to the unit circle, $\left\{z_{\mu},\left|z_{\mu}\right|^{-1} e^{j \arg \left(z_{\mu}\right)}\right\}_{\mu=0}^{N_{t}-1}$, or the $N_{t}$ pairwise roots of $f^{r}(g)=0$ whose imaginary parts have the smallest values, $\left\{\mathfrak{R}\left(g_{\mu}\right) \pm\right.$ $\left.j \mathfrak{J}\left(g_{\mu}\right)\right\}_{\mu=0}^{N_{t}-1}$. Due to the influence of noise, the so-obtained $N_{t}$ 
pairwise roots of $f^{c}(z)=0$ or $f^{r}(g)=0$ may not correspond to $\left\{\beta_{\mu}\right\}_{\mu=0}^{N_{t}-1}$ at low signal-to-noise ratio (SNR). To reduce the influence of noise, we set a threshold $\lambda_{\text {th }}$ at low SNR and compare $\Lambda^{c}\left(z_{\mu}\right)$ or $\Lambda^{r}\left(\mathfrak{R}\left(g_{\mu}\right)\right)$ with $\lambda_{\text {th. }}$. The threshold $\lambda_{\text {th }}$ can be determined through simulation and is often a small value around zero. If $\Lambda^{c}\left(z_{\mu}\right)$ or $\Lambda^{r}\left(\mathfrak{R}\left(g_{\mu}\right)\right)$ exceeds $\lambda_{\text {th }}$, we find the $N_{t}$ pairwise roots of $f^{c}(z)=0$ or $f^{r}(g)=0$ whose corresponding values of $\Lambda^{c}(z)$ or $\Lambda^{r}(\mathfrak{R}(g))$ are the smallest. If $\Lambda^{c}\left(z_{\mu}\right)$ or $\Lambda^{r}\left(\mathfrak{R}\left(g_{\mu}\right)\right)$ is less than $\lambda_{\text {th }}$, we directly use the above obtained $N_{t}$ pairwise roots which are closest to the unit circle or whose imaginary parts have the smallest values. Then, we can readily estimate $\left\{\beta_{\mu}\right\}_{\mu=0}^{N_{t}-1}$ from the $N_{t}$ pairwise roots $\left\{z_{\mu},\left|z_{\mu}\right|^{-1} e^{j \arg \left(z_{\mu}\right)}\right\}_{\mu=0}^{N_{t}-1}$ or $\left\{\mathfrak{R}\left(g_{\mu}\right) \pm j \mathfrak{J}\left(g_{\mu}\right)\right\}_{\mu=0}^{N_{t}-1}$ as follows

$$
\begin{gathered}
\hat{\beta}_{\mu}=\left(Q / 2 / \pi \times \arg \left(z_{\mu}\right)\right)_{Q}, \\
\hat{\beta}_{\mu}=\left(Q / \pi \times \operatorname{acot}\left[\mathfrak{R}\left(g_{\mu}\right)\right]\right)_{Q} .
\end{gathered}
$$

Let $\varepsilon_{i}$ and $\varepsilon_{f}$ denote the ICFO and FCFO, respectively. Then, $\beta_{\mu}$ can be decomposed as $\beta_{\mu}=\varepsilon_{i}+\varepsilon_{f}+i_{\mu}$. Hence, by imposing proper design condition on our CBTS training sequences, we can estimate $\varepsilon_{i}$ and $\varepsilon_{f}$ from $\left\{\hat{\beta}_{\mu}\right\}_{\mu=0}^{N_{t}-1}$. Define

$$
\varepsilon_{f, \mathrm{th}}=\frac{1}{N_{t}} \sum_{\mu=0}^{N_{t}-1}\left\{\left|\hat{\beta}_{\mu}-\operatorname{round}\left(\hat{\beta}_{\mu}\right)\right|\right\} .
$$

To avoid the ambiguous estimation when $\varepsilon_{f}$ is near 0 or $\pm 1 / 2$, we set $i_{\mu}^{\prime}=\left(\text { floor }\left(\hat{\beta}_{\mu}\right)\right)_{Q}$ if $\varepsilon_{f, \text { th }}$ exceeds $1 / 4$. Or else we set $i_{\mu}^{\prime}=$ $\left(\operatorname{round}\left(\hat{\beta}_{\mu}\right)\right)_{Q}$. Then, the FCFO can be immediately estimated as follows

$$
\hat{\varepsilon}_{f}=\frac{1}{N_{t}} \sum_{\mu=0}^{N_{t}-1}\left\{\hat{\beta}_{\mu}-i_{\mu}^{\prime}\right\} .
$$

Let $\boldsymbol{l}$ denote the pilot location vector which is given by $\boldsymbol{l}=$ $\sum_{\mu=0}^{N_{t}-1} \boldsymbol{e}_{Q}^{i_{\mu}}$. To ensure the identifiability of the ICFO estimation, we impose the following condition on $l$

$$
\left(\mathbf{1}_{Q}-\boldsymbol{l}\right)^{T} \boldsymbol{l}^{(q)}>0, \forall q \in\{1,2, \cdots, Q-1\} .
$$

Define $\boldsymbol{l}^{\prime}=\sum_{\mu=0}^{N_{t}-1} \boldsymbol{e}_{Q}^{i_{\mu}^{\prime}}$. Then, the ICFO can be uniquely estimated as follows

$$
\hat{\varepsilon}_{i}=\underset{-Q / 2<q \leq Q / 2}{\arg \max }\left\{\left(\boldsymbol{l}^{\prime}\right)^{T} \boldsymbol{l}^{(q)}\right\} .
$$

Note that the approach in this paper can be applied to the relatively benign channel environments. For the bad channel environments where the SNR is often around or lower than $0 \mathrm{~dB}$, we can still use the approach in [13] [14] whose ICFO estimator is very robust against noise.

\section{B. Analysis of the Direct Rooting Approach and the Derivative Rooting Approach}

It has been shown recently in [15] that CFO estimation via polynomial rooting from the first-order derivative of the cost function is superior to that via polynomial rooting from the cost function in blind single antenna OFDM systems. Note that the derivative rooting approach in [15] can be applied directly in our considered MIMO OFDM systems.

Let $\boldsymbol{E}_{\boldsymbol{W}}$ denote the $Q \times\left(Q-N_{t}\right)$ real matrix which contains the unitary eigen-vectors spanning the noise subspace of $\hat{\boldsymbol{R}}_{\boldsymbol{Y} \boldsymbol{Y}}$. Then, we have

$$
\boldsymbol{E}_{\boldsymbol{X}} \boldsymbol{E}_{\boldsymbol{X}}^{T}+\boldsymbol{E}_{W} \boldsymbol{E}_{W}^{T}=\boldsymbol{I}_{Q}
$$

Besides, since $\boldsymbol{L}$ is a column conjugate symmetric matrix, we also have

$$
\boldsymbol{J} \boldsymbol{L}=\boldsymbol{L}^{*}
$$

Hence, $f^{c}(z)$ and $f^{r}(g)$ can be further expressed as follows

$$
\begin{aligned}
& f^{c}(z)=\left[\boldsymbol{k}^{c}(z)\right]^{T} \boldsymbol{k}^{c}(z), \\
& f^{r}(g)=\left[\boldsymbol{k}^{r}(g)\right]^{T} \boldsymbol{k}^{r}(g),
\end{aligned}
$$

where

$$
\begin{gathered}
\boldsymbol{k}^{c}(z)=\boldsymbol{E}_{\boldsymbol{W}}^{T} \boldsymbol{L}^{H} \boldsymbol{a}(z), \\
\boldsymbol{k}^{r}(g)=\boldsymbol{E}_{\boldsymbol{W}}^{T} \boldsymbol{L}^{H} \boldsymbol{\Phi} \boldsymbol{a}(g) .
\end{gathered}
$$

Correspondingly, $\Lambda^{c}(z)$ and $\Lambda^{r}(g)$ can be expressed as follows

$$
\begin{gathered}
\Lambda^{c}(z)=z^{1-Q} \cdot\left[\boldsymbol{k}^{c}(z)\right]^{T} \boldsymbol{k}^{c}(z), \\
\Lambda^{r}(g)=\left(g^{2}+1\right)^{1-Q} \cdot\left[\boldsymbol{k}^{r}(g)\right]^{T} \boldsymbol{k}^{r}(g) .
\end{gathered}
$$

Taking the first-order derivative of $f^{c}(z)$ and $f^{r}(g)$ with respect to $z$ and $g$ respectively, we immediately establish

$$
\begin{aligned}
& \left(f^{c}(z)\right)^{\prime}=2\left[\left(\boldsymbol{k}^{c}(z)\right)^{T}\right]^{\prime} \boldsymbol{k}^{c}(z), \\
& \left(f^{r}(g)\right)^{\prime}=2\left[\left(\boldsymbol{k}^{r}(g)\right)^{T}\right]^{\prime} \boldsymbol{k}^{r}(g) .
\end{aligned}
$$

Note that $\Lambda^{c}(z), f^{c}(z)$ and $\left(f^{c}(z)\right)^{\prime}$ have the common polynomial factor $\boldsymbol{k}^{c}(z)$, while $\Lambda^{r}(g), f^{r}(g)$ and $\left(f^{r}(g)\right)^{\prime}$ have the common polynomial factor $\boldsymbol{k}^{r}(g)$.

Let $z_{\mu}=e^{j 2 \pi \beta_{\mu} / Q}, g_{\mu}=\cot \left(\pi \beta_{\mu} / Q\right)$. In the ideal case without considering noise, we have

$$
f^{c}\left(z_{\mu}\right)=0, f^{r}\left(g_{\mu}\right)=0 .
$$

Besides, $\Lambda^{c}(z)$ and $f^{c}(z)$ can also be written into the following equivalent forms [13]

$$
\begin{gathered}
\Lambda^{c}(z)=\left[\boldsymbol{k}^{c}(z)\right]^{H} \boldsymbol{k}^{c}(z), \\
f^{c}(z)=z^{Q-1} \cdot\left[\boldsymbol{k}^{c}(z)\right]^{H} \boldsymbol{k}^{c}(z) .
\end{gathered}
$$

We can see from (18) and (25) that $f^{c}\left(z_{\mu}\right)=0$ and $f^{r}\left(g_{\mu}\right)=0$ hold if and only if

$$
\boldsymbol{k}^{c}\left(z_{\mu}\right)=\mathbf{0}_{Q-N_{t}}, \boldsymbol{k}^{r}\left(g_{\mu}\right)=\mathbf{0}_{Q-N_{t}} .
$$

Due to the common polynomial factors $\boldsymbol{k}^{c}(z)$ and $\boldsymbol{k}^{r}(g)$, it follows immediately from (26) that

$$
\begin{aligned}
& \left(f^{c}\left(z_{\mu}\right)\right)^{\prime}=0, \Lambda^{c}\left(z_{\mu}\right)=0, \\
& \left(f^{r}\left(g_{\mu}\right)\right)^{\prime}=0, \Lambda^{r}\left(g_{\mu}\right)=0 .
\end{aligned}
$$

From the above analysis, we can see that the roots from the direct rooting and derivative rooting approaches correspond to the same ones which make $\boldsymbol{k}^{c}\left(z_{\mu}\right)$ or $\boldsymbol{k}^{r}\left(g_{\mu}\right)$ equal $\mathbf{0}_{Q-N_{t}}$ in the absence of noise or approximately equal $\mathbf{0}_{Q-N_{t}}$ in the 
presence of noise. In this way, we can say that the derivative rooting approach is equivalent to the direct rooting approach for the considered MIMO case, which will be verified through simulation results in the next section. Actually, the above mentioned two rooting approaches are also equivalent for the blind single antenna case in [15], whose analytical result is omitted here due to space limitation.

\section{Computational Complexity}

For description convenience, we only consider the real polynomial case here. For the direct rooting approach, the $N_{t}$ pairwise roots of $f^{r}(g)=0$ whose imaginary parts have the smallest values match well with the ones whose corresponding values of $\Lambda^{r}(\mathfrak{R}(g))$ are the smallest at high SNR completely and at low SNR mostly, which can be easily verified through simulations. Therefore, the direct rooting approach only needs to calculate the values of the cost function $\Lambda^{r}(\mathfrak{R}(g))$ with respect to the roots of $f^{r}(g)=0$ at low SNR occasionally. Hence, the computational load of the direct rooting approach mainly involves the eigen-decomposition of $\hat{\boldsymbol{R}}_{\boldsymbol{Y} Y}$ and the rootcalculation of $f^{r}(g)=0$, which require $9 Q^{3}$ and $64 / 3 \cdot(Q-1)^{3}$ real additions or multiplications [17] [18], respectively. While for the derivative rooting approach, although its computation is decreased slightly due to the degree reduction of $\left(f^{r}(g)\right)^{\prime}$ in comparison with $f^{r}(g)$, it still needs an additional complicated calculation of the values of the cost function $\Lambda^{r}(\mathfrak{R}(g))$ with respect to the roots of $\left(f^{r}(g)\right)^{\prime}=0$, which requires $4(Q-3 / 2)\left(Q-N_{t}\right) Q(Q+1)$ real additions or multiplications. Therefore, the direct rooting approach has lower computational complexity than the derivative rooting approach. Besides, since the direct rooting approach does not need the DFT operation, its complexity is also lower than that in [13], [14].

\section{Simulation Results}

We provide simulations to validate the theoretical analysis and also to evaluate the performance of the proposed CFO estimators with the CBTS training sequences. In the simulations, we use a MIMO OFDM system with $N=1024$ subcarriers, $N_{t}=3$ transmit antennas and $N_{r}=2$ receive antennas. The channels are assumed independent and have 4 uncorrelated Rayleigh fading taps each. The relative propagation delays are chosen equal to $\{0,0.1,0.2,0.4\} \mu \mathrm{s}$, and the variances of the taps are $\{0,-9.7,-19.2,-22.8\} \mathrm{dB}$. The normalized CFO $\varepsilon$ is generated within the range $(-Q / 2, Q / 2]$. For description convenience, we refer to the CFO estimator in [13] [14], the $\mathrm{CFO}$ estimators via derivative rooting and direct rooting proposed in this paper as Estimator A, Estimator B and Estimator C, respectively.

As was pointed out in [19] [20], the extended Miller and Chang bound (EMCB) can be tighter than the Cramer-Rao bound (CRB). In the following, we adopt the EMCB to benchmark the performance of the considered $\mathrm{CFO}$ estimators via polynomial rooting. The EMCB is obtained by averaging the snapshot CRB over independent channel realizations [19] [21] as follows

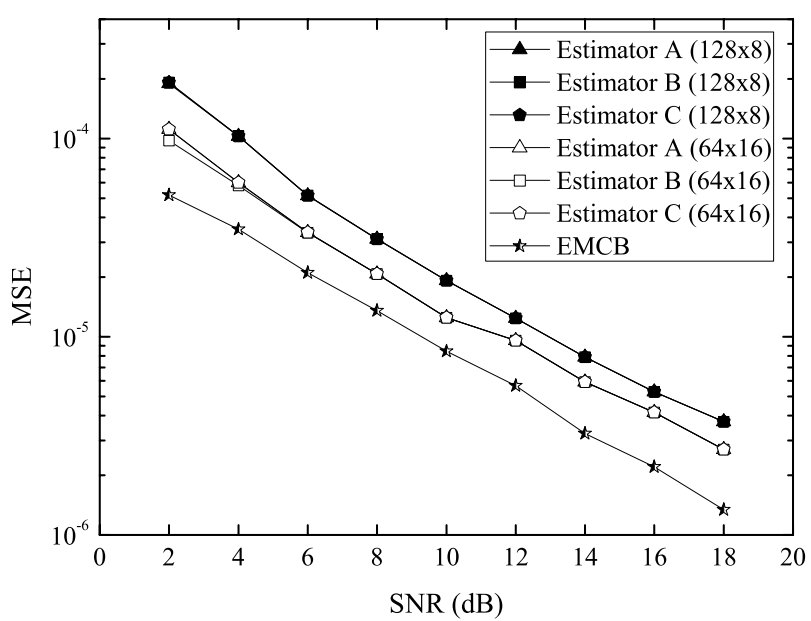

Fig. 1. MSE performance of the CFO estimators via complex polynomial rooting with $P=128, Q=8$ and $P=64, Q=16$.

$$
\begin{aligned}
\mathrm{EMCB}_{\varepsilon} & = \\
\mathrm{E} & \left\{\frac{N \sigma_{w}^{2}}{8 \pi^{2} \boldsymbol{h}^{H} \boldsymbol{X}^{H} \mathcal{B}\left[\boldsymbol{I}_{N_{r} N}-\boldsymbol{X}\left(\boldsymbol{X}^{H} \boldsymbol{X}\right)^{-1} \boldsymbol{X}^{H}\right] \mathcal{B} \boldsymbol{X} \boldsymbol{h}}\right\},
\end{aligned}
$$

where

$$
\begin{gathered}
\boldsymbol{X}=\boldsymbol{I}_{N_{r}} \otimes \boldsymbol{S}, \\
\mathcal{B}=\boldsymbol{I}_{N_{r}} \otimes \operatorname{diag}\left\{\left[N_{g}, N_{g}+1, \cdots, N_{g}+N-1\right]^{T}\right\} .
\end{gathered}
$$

We resort to Monte Carlo simulation for its evaluation in this paper.

Fig. 1 shows the mean square error (MSE) performance of the considered three $\mathrm{CFO}$ estimators via complex polynomial rooting with $P=128, Q=8$ and $P=64, Q=16$. Fig. 2 presents the MSE performance of the CFO estimators via real polynomial rooting. Also included in the two figures is the EMCB. We can see that Estimator A, B and C via complex polynomial rooting have almost the same MSE performance. While for the real polynomial case, Estimator C (i.e., the estimator via direct rooting) slightly outperforms the other two estimators. Actually, in the ideal case without considering noise, the three estimators via real polynomial rooting also have almost the same MSE performance. We can see that the simulation results support the theoretical results very well, except a slight mismatch at low SNR in Fig. 2. This could be due to different outlier statistics and is under investigation.

In Fig. 3, we show the MSE performance of the CFO estimator in [13] [14] via complex and real polynomial rooting at low SNR. It can be seen that the estimation performance is satisfactory and acceptable even when the SNR is quite lower than $0 \mathrm{~dB}$. Therefore, in the environments which are affected greatly by the noise, we can employ the estimator in [13] [14].

\section{CONCLUSIONS}

In this paper, we have presented a simplified $\mathrm{CFO}$ estimator via direct polynomial rooting for MIMO OFDM systems by designing the training sequences. We have analyzed the $\mathrm{CFO}$ 


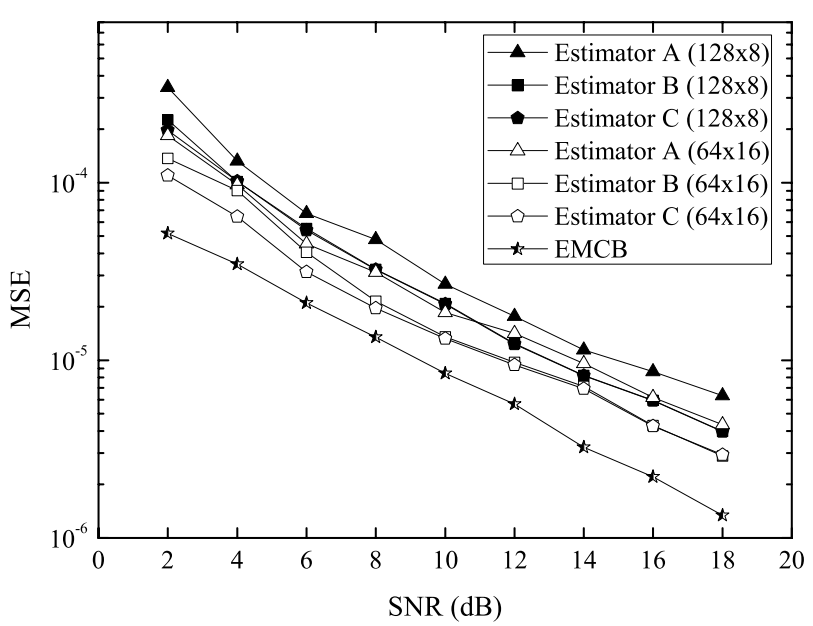

Fig. 2. MSE performance of the CFO estimators via real polynomial rooting with $P=128, Q=8$ and $P=64, Q=16$.

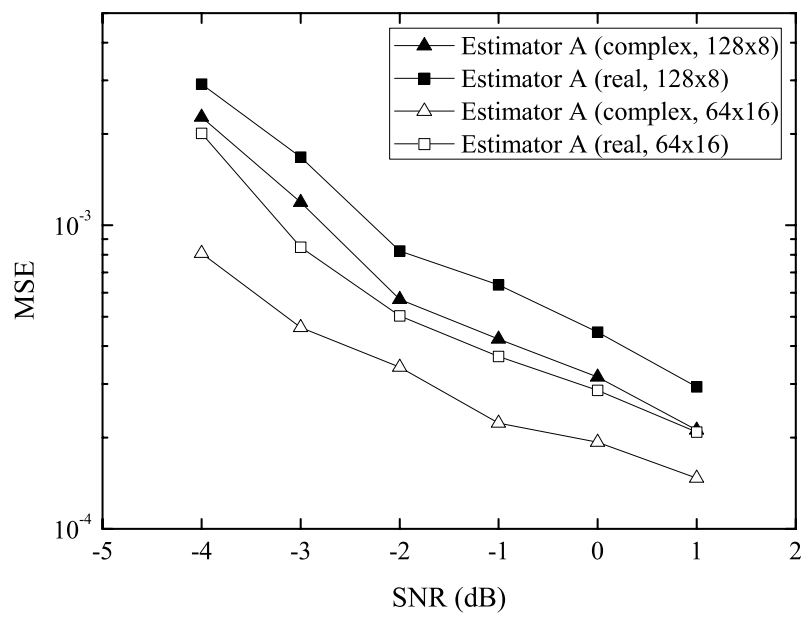

Fig. 3. MSE performance of the CFO estimator in [13] [14] via complex and real polynomial rooting at low SNR.

estimation via direct polynomial rooting and via derivative polynomial rooting. Our analytical results show that the two approaches are equivalent and they are well supported by the simulation results.

\section{ACKNOWLEDGMENT}

This work was supported in part by National Natural Science Foundation of China under Grant 60496310 and 60702028, the China High-Tech 863 Project under Grant 2007AA01Z200, and in part by the Erik Jonsson School
Research Excellence Initiative, the University of Texas at Dallas, USA.

\section{REFERENCES}

[1] T. Pollet, M. V. Bladel, and M. Moeneclaey, "BER sensitivity of OFDM systems to carrier frequency offset and Wiener phase noise," IEEE Trans. Commun., vol. 43, pp. 191-193, Feb./Mar./Apr. 1995.

[2] J. van de Beek, M. Sandell, and P. O. Borjesson, "ML estimation of time and frequency offset in OFDM systems," IEEE Trans. Signal Processing, vol. 45, pp. 1800-1805, July 1997.

[3] U. Tureli, H. Liu, and M. D. Zoltowski, "OFDM blind carrier offset estimation: ESPRIT," IEEE Trans. Commun., vol. 48, pp. 1459-1461, Sept. 2000.

[4] P. Moose, "A technique for orthogonal frequency division multiplexing frequency offset correction," IEEE Trans. Commun., vol. 42, pp. 2908 2914, Oct. 1994.

[5] T. Schmidl and D. C. Cox, "Robust frequency and timing synchronization for OFDM," IEEE Trans. Commun., vol. 45, pp. 1613-1621, Dec. 1997.

[6] M. Morelli and U. Mengali, "An improved frequency offset estimator for OFDM applications," IEEE Commun. Lett., vol. 3, pp. 75-77, Mar 1999.

[7] H. Minn, V. K. Bhargava, and K. B. Letaief, "A robust timing and frequency synchronization for OFDM systems," IEEE Trans. Wireless Commun., vol. 2, no. 4, pp. 822-839, July 2003.

[8] D. Huang and K. B. Letaief, "Carrier frequency offset estimation for OFDM systems using null subcarriers," IEEE Trans. Commun., vol. 54 no. 5, pp. 813-823, May 2006.

[9] A. N. Mody and G. L. Stuber, "Synchronization for MIMO OFDM systems," in Proc. IEEE Globecom'01, vol. 1, Nov. 2001, pp. 509-513.

[10] O. Besson and P. Stoica, "On parameter estimation of MIMO flatfading channels with frequency offsets," IEEE Trans. Signal Processing, vol. 51, no. 3, pp. 602-613, Mar. 2003.

[11] X. Ma, M. K. Oh, G. B. Giannakis, and D. J. Park, "Hopping pilots for estimation of frequency-offset and multi-antenna channels in MIMO OFDM," IEEE Trans. Commun., vol. 53, no. 1, pp. 162-172, Jan. 2005.

[12] F. Simoens and M. Moeneclaey, "Reduced complexity data-aided and code-aided frequency offset estimation for flat-fading MIMO channels,' IEEE Trans. Wireless Commun., vol. 5, no. 6, pp. 1558-1567, June 2006

[13] Y. X. Jiang, X. Q. Gao, X. H. You, and W. Heng, "Training sequence assisted frequency offset estimation for MIMO OFDM," in Proc. IEEE ICC'O6, vol. 12, June 2006, pp. 5371-5376.

[14] Y. X. Jiang, H. Minn, X. Q. Gao, X. H. You, and Y. Li, "Frequency offset estimation and training sequence design for MIMO OFDM," to appear in IEEE Trans. Wireless Commun.

[15] F. Gao and A. Nallanathan, "Blind maximum likelihood CFO estimation for OFDM systems via polynomial rooting," IEEE Signal Processing Lett., vol. 13, no. 2, pp. 73-76, Feb. 2006.

[16] D. Chu, "Polyphase codes with good periodic correlation properties,' IEEE Trans. Inform. Theory, vol. 18, pp. 531-532, July 1972.

[17] W. H. Press, Numerical Recipes in C++: the Art of Scientific Computing. Cambridge: Cambridge University Press, 2002.

[18] G. H. Golub and C. F. Van Loan, Matrix Computations. The John Hopkins University Press, 1996.

[19] F. Gini and R. Reggiannini, "On the use of Cramer-Rao-like bounds in the presence of random nuisance parameters," IEEE Trans. Commun. vol. 48 , no. 12 , pp. $2120-2126$, Dec. 2000.

[20] H. Minn, X. Fu, and V. K. Bhargava, "Optimal periodic training signal for frequency offset estimation in frequency-selective fading channels," IEEE Trans. Commun., vol. 54, no. 6, pp. 1081-1096, June 2006.

[21] S. M. Kay, Fundamentals of Statistical Signal Processing: Estimation Theory, Englewood Cliffs ed. NJ: Prentical-Hall, 1993. 